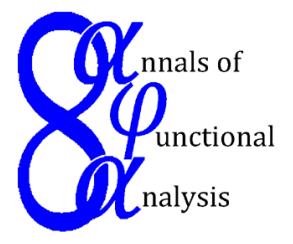

Ann. Funct. Anal. 7 (2016), no. 1, 118-126

http://dx.doi.org/10.1215/20088752-3429463

ISSN: 2008-8752 (electronic)

http://projecteuclid.org/afa

\title{
LIFTING PROBLEMS FOR NORMED SPACES
}

\author{
NIELS GRØNBæK \\ Dedicated to Professor Anthony To-Ming Lau \\ Communicated by D. Leung
}

\begin{abstract}
A classical theorem of G. Köthe states that the Banach spaces $X$ with the property that all bounded linear maps $X \rightarrow Y$ into an arbitrary Banach space $Y$ can be lifted with respect to bounded linear surjections onto $Y$ are up to topological linear isomorphism precisely the spaces $\ell^{1}(A)$. We extend this result to the category of normed linear spaces and bounded linear maps. This answers a question raised by A. Ya. Helemskiı̌.
\end{abstract}

\section{InTRODUCTION}

Extension and lifting problems are at the core of the theory of topological vector spaces and continuous linear maps. We start by considering these problems in the setting of Banach spaces and bounded linear maps. Let $F$ be a Banach space with a closed subspace $E$. An extension problem is a diagram

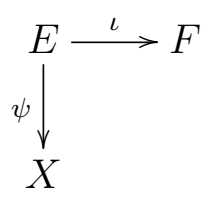

and a solution is a bounded linear map $\tilde{\psi}: F \rightarrow X$ such that $\psi=\iota \tilde{\psi}$. A rather straightforward application of Hahn-Banach's theorem yields the result that if $X=\ell^{\infty}(A)$, then all extension problems can be solved. In fact, we have solutions

Copyright 2016 by the Tusi Mathematical Research Group.

Received Mar. 20, 2015; Accepted Jun. 9, 2015.

2010 Mathematics Subject Classification. Primary 46B20; Secondary 46B03, 46M10.

Keywords. lifting problems, projectives, noncomplete normed spaces, Hahn-Banach theorem. 


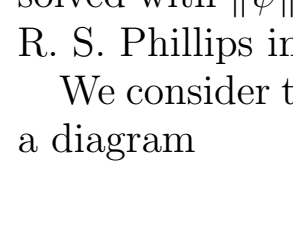

Ann. Funct. Anal. 7 (2016), no. 1, 118-126

http://dx.doi.org/10.1215/20088752-3429463

ISSN: 2008-8752 (electronic)

http://projecteuclid.org/afa

\title{
LIFTING PROBLEMS FOR NORMED SPACES
}

\author{
NIELS GRØNBæK \\ Dedicated to Professor Anthony To-Ming Lau \\ Communicated by D. Leung
}

\begin{abstract}
A classical theorem of G. Köthe states that the Banach spaces $X$ with the property that all bounded linear maps $X \rightarrow Y$ into an arbitrary Banach space $Y$ can be lifted with respect to bounded linear surjections onto $Y$ are up to topological linear isomorphism precisely the spaces $\ell^{1}(A)$. We extend this result to the category of normed linear spaces and bounded linear maps. This answers a question raised by A. Ya. Helemskiı̌.
\end{abstract}

\section{InTRODUCTION}

Extension and lifting problems are at the core of the theory of topological vector spaces and continuous linear maps. We start by considering these problems in the setting of Banach spaces and bounded linear maps. Let $F$ be a Banach space with a closed subspace $E$. An extension problem is a diagram

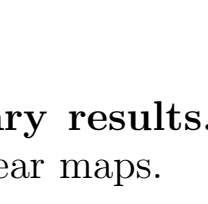

and a solution is a bounded linear map $\tilde{\psi}: F \rightarrow X$ such that $\psi=\iota \tilde{\psi}$. A rather straightforward application of Hahn-Banach's theorem yields the result that if $X=\ell^{\infty}(A)$, then all extension problems can be solved. In fact, we have solutions

Copyright 2016 by the Tusi Mathematical Research Group.

Received Mar. 20, 2015; Accepted Jun. 9, 2015.

2010 Mathematics Subject Classification. Primary 46B20; Secondary 46B03, 46M10.

Keywords. lifting problems, projectives, noncomplete normed spaces, Hahn-Banach theorem. 


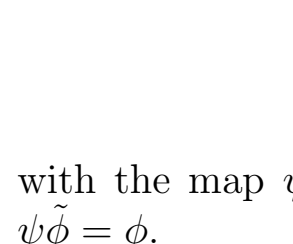

Ann. Funct. Anal. 7 (2016), no. 1, 118-126

http://dx.doi.org/10.1215/20088752-3429463

ISSN: 2008-8752 (electronic)

http://projecteuclid.org/afa

\title{
LIFTING PROBLEMS FOR NORMED SPACES
}

\author{
NIELS GRØNBæK \\ Dedicated to Professor Anthony To-Ming Lau \\ Communicated by D. Leung
}

\begin{abstract}
A classical theorem of G. Köthe states that the Banach spaces $X$ with the property that all bounded linear maps $X \rightarrow Y$ into an arbitrary Banach space $Y$ can be lifted with respect to bounded linear surjections onto $Y$ are up to topological linear isomorphism precisely the spaces $\ell^{1}(A)$. We extend this result to the category of normed linear spaces and bounded linear maps. This answers a question raised by A. Ya. Helemskiı̌.
\end{abstract}

\section{InTRODUCTION}

Extension and lifting problems are at the core of the theory of topological vector spaces and continuous linear maps. We start by considering these problems in the setting of Banach spaces and bounded linear maps. Let $F$ be a Banach space with a closed subspace $E$. An extension problem is a diagram

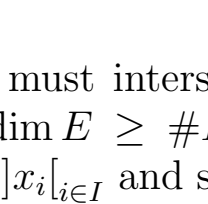

and a solution is a bounded linear map $\tilde{\psi}: F \rightarrow X$ such that $\psi=\iota \tilde{\psi}$. A rather straightforward application of Hahn-Banach's theorem yields the result that if $X=\ell^{\infty}(A)$, then all extension problems can be solved. In fact, we have solutions

Copyright 2016 by the Tusi Mathematical Research Group.

Received Mar. 20, 2015; Accepted Jun. 9, 2015.

2010 Mathematics Subject Classification. Primary 46B20; Secondary 46B03, 46M10.

Keywords. lifting problems, projectives, noncomplete normed spaces, Hahn-Banach theorem. 


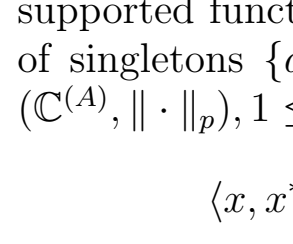

Ann. Funct. Anal. 7 (2016), no. 1, 118-126

http://dx.doi.org/10.1215/20088752-3429463

ISSN: 2008-8752 (electronic)

http://projecteuclid.org/afa

\title{
LIFTING PROBLEMS FOR NORMED SPACES
}

\author{
NIELS GRØNBæK \\ Dedicated to Professor Anthony To-Ming Lau \\ Communicated by D. Leung
}

\begin{abstract}
A classical theorem of G. Köthe states that the Banach spaces $X$ with the property that all bounded linear maps $X \rightarrow Y$ into an arbitrary Banach space $Y$ can be lifted with respect to bounded linear surjections onto $Y$ are up to topological linear isomorphism precisely the spaces $\ell^{1}(A)$. We extend this result to the category of normed linear spaces and bounded linear maps. This answers a question raised by A. Ya. Helemskiı̌.
\end{abstract}

\section{InTRODUCTION}

Extension and lifting problems are at the core of the theory of topological vector spaces and continuous linear maps. We start by considering these problems in the setting of Banach spaces and bounded linear maps. Let $F$ be a Banach space with a closed subspace $E$. An extension problem is a diagram

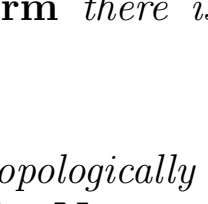

and a solution is a bounded linear map $\tilde{\psi}: F \rightarrow X$ such that $\psi=\iota \tilde{\psi}$. A rather straightforward application of Hahn-Banach's theorem yields the result that if $X=\ell^{\infty}(A)$, then all extension problems can be solved. In fact, we have solutions

Copyright 2016 by the Tusi Mathematical Research Group.

Received Mar. 20, 2015; Accepted Jun. 9, 2015.

2010 Mathematics Subject Classification. Primary 46B20; Secondary 46B03, 46M10.

Keywords. lifting problems, projectives, noncomplete normed spaces, Hahn-Banach theorem. 


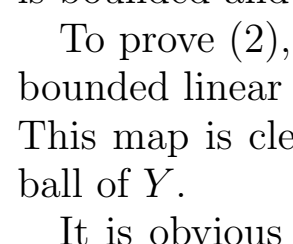

Ann. Funct. Anal. 7 (2016), no. 1, 118-126

http://dx.doi.org/10.1215/20088752-3429463

ISSN: 2008-8752 (electronic)

http://projecteuclid.org/afa

\title{
LIFTING PROBLEMS FOR NORMED SPACES
}

\author{
NIELS GRØNBæK \\ Dedicated to Professor Anthony To-Ming Lau \\ Communicated by D. Leung
}

\begin{abstract}
A classical theorem of G. Köthe states that the Banach spaces $X$ with the property that all bounded linear maps $X \rightarrow Y$ into an arbitrary Banach space $Y$ can be lifted with respect to bounded linear surjections onto $Y$ are up to topological linear isomorphism precisely the spaces $\ell^{1}(A)$. We extend this result to the category of normed linear spaces and bounded linear maps. This answers a question raised by A. Ya. Helemskiı̌.
\end{abstract}

\section{InTRODUCTION}

Extension and lifting problems are at the core of the theory of topological vector spaces and continuous linear maps. We start by considering these problems in the setting of Banach spaces and bounded linear maps. Let $F$ be a Banach space with a closed subspace $E$. An extension problem is a diagram

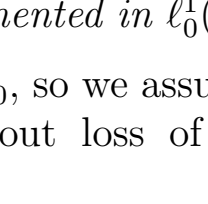

and a solution is a bounded linear map $\tilde{\psi}: F \rightarrow X$ such that $\psi=\iota \tilde{\psi}$. A rather straightforward application of Hahn-Banach's theorem yields the result that if $X=\ell^{\infty}(A)$, then all extension problems can be solved. In fact, we have solutions

Copyright 2016 by the Tusi Mathematical Research Group.

Received Mar. 20, 2015; Accepted Jun. 9, 2015.

2010 Mathematics Subject Classification. Primary 46B20; Secondary 46B03, 46M10.

Keywords. lifting problems, projectives, noncomplete normed spaces, Hahn-Banach theorem. 


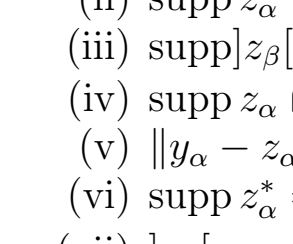

Ann. Funct. Anal. 7 (2016), no. 1, 118-126

http://dx.doi.org/10.1215/20088752-3429463

ISSN: 2008-8752 (electronic)

http://projecteuclid.org/afa

\title{
LIFTING PROBLEMS FOR NORMED SPACES
}

\author{
NIELS GRØNBæK \\ Dedicated to Professor Anthony To-Ming Lau \\ Communicated by D. Leung
}

\begin{abstract}
A classical theorem of G. Köthe states that the Banach spaces $X$ with the property that all bounded linear maps $X \rightarrow Y$ into an arbitrary Banach space $Y$ can be lifted with respect to bounded linear surjections onto $Y$ are up to topological linear isomorphism precisely the spaces $\ell^{1}(A)$. We extend this result to the category of normed linear spaces and bounded linear maps. This answers a question raised by A. Ya. Helemskiı̌.
\end{abstract}

\section{InTRODUCTION}

Extension and lifting problems are at the core of the theory of topological vector spaces and continuous linear maps. We start by considering these problems in the setting of Banach spaces and bounded linear maps. Let $F$ be a Banach space with a closed subspace $E$. An extension problem is a diagram

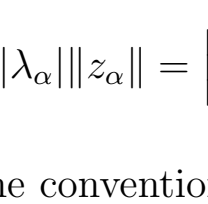

and a solution is a bounded linear map $\tilde{\psi}: F \rightarrow X$ such that $\psi=\iota \tilde{\psi}$. A rather straightforward application of Hahn-Banach's theorem yields the result that if $X=\ell^{\infty}(A)$, then all extension problems can be solved. In fact, we have solutions

Copyright 2016 by the Tusi Mathematical Research Group.

Received Mar. 20, 2015; Accepted Jun. 9, 2015.

2010 Mathematics Subject Classification. Primary 46B20; Secondary 46B03, 46M10.

Keywords. lifting problems, projectives, noncomplete normed spaces, Hahn-Banach theorem. 


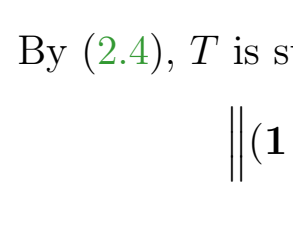

Ann. Funct. Anal. 7 (2016), no. 1, 118-126

http://dx.doi.org/10.1215/20088752-3429463

ISSN: 2008-8752 (electronic)

http://projecteuclid.org/afa

\title{
LIFTING PROBLEMS FOR NORMED SPACES
}

\author{
NIELS GRØNBæK \\ Dedicated to Professor Anthony To-Ming Lau \\ Communicated by D. Leung
}

\begin{abstract}
A classical theorem of G. Köthe states that the Banach spaces $X$ with the property that all bounded linear maps $X \rightarrow Y$ into an arbitrary Banach space $Y$ can be lifted with respect to bounded linear surjections onto $Y$ are up to topological linear isomorphism precisely the spaces $\ell^{1}(A)$. We extend this result to the category of normed linear spaces and bounded linear maps. This answers a question raised by A. Ya. Helemskiı̌.
\end{abstract}

\section{InTRODUCTION}

Extension and lifting problems are at the core of the theory of topological vector spaces and continuous linear maps. We start by considering these problems in the setting of Banach spaces and bounded linear maps. Let $F$ be a Banach space with a closed subspace $E$. An extension problem is a diagram

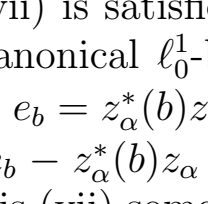

and a solution is a bounded linear map $\tilde{\psi}: F \rightarrow X$ such that $\psi=\iota \tilde{\psi}$. A rather straightforward application of Hahn-Banach's theorem yields the result that if $X=\ell^{\infty}(A)$, then all extension problems can be solved. In fact, we have solutions

Copyright 2016 by the Tusi Mathematical Research Group.

Received Mar. 20, 2015; Accepted Jun. 9, 2015.

2010 Mathematics Subject Classification. Primary 46B20; Secondary 46B03, 46M10.

Keywords. lifting problems, projectives, noncomplete normed spaces, Hahn-Banach theorem. 


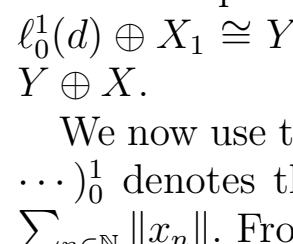

Ann. Funct. Anal. 7 (2016), no. 1, 118-126

http://dx.doi.org/10.1215/20088752-3429463

ISSN: 2008-8752 (electronic)

http://projecteuclid.org/afa

\title{
LIFTING PROBLEMS FOR NORMED SPACES
}

\author{
NIELS GRØNBæK \\ Dedicated to Professor Anthony To-Ming Lau \\ Communicated by D. Leung
}

\begin{abstract}
A classical theorem of G. Köthe states that the Banach spaces $X$ with the property that all bounded linear maps $X \rightarrow Y$ into an arbitrary Banach space $Y$ can be lifted with respect to bounded linear surjections onto $Y$ are up to topological linear isomorphism precisely the spaces $\ell^{1}(A)$. We extend this result to the category of normed linear spaces and bounded linear maps. This answers a question raised by A. Ya. Helemskiı̌.
\end{abstract}

\section{InTRODUCTION}

Extension and lifting problems are at the core of the theory of topological vector spaces and continuous linear maps. We start by considering these problems in the setting of Banach spaces and bounded linear maps. Let $F$ be a Banach space with a closed subspace $E$. An extension problem is a diagram

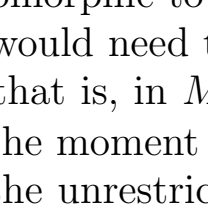

and a solution is a bounded linear map $\tilde{\psi}: F \rightarrow X$ such that $\psi=\iota \tilde{\psi}$. A rather straightforward application of Hahn-Banach's theorem yields the result that if $X=\ell^{\infty}(A)$, then all extension problems can be solved. In fact, we have solutions

Copyright 2016 by the Tusi Mathematical Research Group.

Received Mar. 20, 2015; Accepted Jun. 9, 2015.

2010 Mathematics Subject Classification. Primary 46B20; Secondary 46B03, 46M10.

Keywords. lifting problems, projectives, noncomplete normed spaces, Hahn-Banach theorem. 


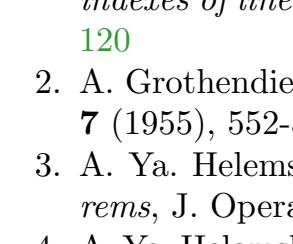

Ann. Funct. Anal. 7 (2016), no. 1, 118-126

http://dx.doi.org/10.1215/20088752-3429463

ISSN: 2008-8752 (electronic)

http://projecteuclid.org/afa

\title{
LIFTING PROBLEMS FOR NORMED SPACES
}

\author{
NIELS GRØNBæK \\ Dedicated to Professor Anthony To-Ming Lau \\ Communicated by D. Leung
}

\begin{abstract}
A classical theorem of G. Köthe states that the Banach spaces $X$ with the property that all bounded linear maps $X \rightarrow Y$ into an arbitrary Banach space $Y$ can be lifted with respect to bounded linear surjections onto $Y$ are up to topological linear isomorphism precisely the spaces $\ell^{1}(A)$. We extend this result to the category of normed linear spaces and bounded linear maps. This answers a question raised by A. Ya. Helemskiı̌.
\end{abstract}

\section{InTRODUCTION}

Extension and lifting problems are at the core of the theory of topological vector spaces and continuous linear maps. We start by considering these problems in the setting of Banach spaces and bounded linear maps. Let $F$ be a Banach space with a closed subspace $E$. An extension problem is a diagram

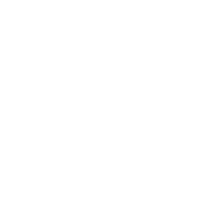

and a solution is a bounded linear map $\tilde{\psi}: F \rightarrow X$ such that $\psi=\iota \tilde{\psi}$. A rather straightforward application of Hahn-Banach's theorem yields the result that if $X=\ell^{\infty}(A)$, then all extension problems can be solved. In fact, we have solutions

Copyright 2016 by the Tusi Mathematical Research Group.

Received Mar. 20, 2015; Accepted Jun. 9, 2015.

2010 Mathematics Subject Classification. Primary 46B20; Secondary 46B03, 46M10.

Keywords. lifting problems, projectives, noncomplete normed spaces, Hahn-Banach theorem. 